\title{
Wer wird ein guter Chefarzt* sein?
}

\section{Marco Mumenthaler}

Der Autor war langjähriger Ordinarius für Neurologie an der Medizinischen Fakultät der Universität Bern und Direktor der Neurologischen Universitätsklinik Bern. Nach der Emeritierung im Jahr 1990 war er privatärztlich als Fachneurologe in Zürich tätig, wo er sich auch im Unterricht für Studierende sowie in der Fortbildung für Ärzte engagierte.
Vor 60 Jahren trat ich meine erste Stelle als jüngster Assistenzarzt in einer Universitätsklinik an. Während weiterer 40 Jahre war ich an Universitätskliniken tätig und war dabei fast 30 Jahre Chefarzt und Mitglied einer Medizinischen Fakultät. Seither habe ich als Arzt ausserhalb eines Spitals praktiziert. In unterschiedlichen Funktionen und aus unterschiedlichen Blickwinkeln habe ich Chefärztinnen und Chefärzte in ihrem Wirken und in ihrer Auswirkung erlebt.

Ich habe über Verschiedenstes mich mündlich und schriftlich geäussert. Einen Plan hatte ich Jahr um Jahr hinausgeschoben, diesen möchte ich mit diesen Zeilen gegen Ende meines Lebens doch noch verwirklichen: Ich möchte versuchen zu definieren, was ein guter Chefarzt - ja, allgemeiner noch: was ein guter Vorgesetzter - ist. Dies möchte ich aus verschiedenen Gründen noch zu Papier bringen. Einmal, weil ich allzu oft erlebt habe, wie junge Ärztinnen und Ärzte sich vergeblich nach einem guten Chef sehnen. Andererseits, weil ich immer wieder erlebt habe, wie oft kleine, vermeidbare Fehler einem Vorgesetzten die Chance verderben, ein guter Chef zu sein. Hierbei stütze ich mich auf bei Kollegen Beobachtetes, auch auf mein eigenes Wirken, aber noch viel mehr auf die Reflexion begangener eigener Fehler.

Verwaltungsräte, Stiftungsräte, Wahlbehörden, politische und andere Instanzen wählen Menschen für bestimmte Funktionen. Daraus ergibt sich ein
Er muss eine überdurchschnittliche Fachkompetenz in dem von ihm vertretenen Bereich der Medizin haben. Diese muss jedoch gerade heutzutage noch weiter spezifiziert und differenziert werden. Die vertieften Kenntnisse innerhalb eines bestimmten Bereichs und damit die Subspezialisierung innerhalb der einzelnen Bereiche ist so weit fortgeschritten, dass ein Einzelner unmöglich in jedem Subbereich seines Hauptfaches der Beste sein kann. Dies gilt besonders für die früher grossen Fächer wie die Innere Medizin und die Chirurgie, trifft aber in zunehmendem Masse auch für verschiedene kleinere Fachbereiche wie zum Beispiel die Neurologie oder die Orthopädie zu. Ein Chef muss also fähig sein, neben sich andere qualifizierte Fachleute wirken zu lassen, die in ihrem speziellen Bereich besser sind als er. Er darf diesen nicht aus Neid eine angemessene Entfaltung verunmöglichen. Ja, er muss ihnen sogar im Rahmen des betriebswirtschaftlich Machbaren eine befriedigende Position mit dem nötigen Mass an Selbständigkeit verschaffen. Er muss ihnen im Rahmen des finanziell Möglichen auch ein angemessenes Einkommen vermitteln Er muss aber auch die Förderung und die Beförderung eines solchen Mitarbeiters anstreben und in seine Planung einbeziehen. Dies setzt eine gefestigte Persönlichkeit voraus, deren Selbstbewusstsein nicht von der Ausübung einer Macht abhängt und bei dem Toleranz stärker als Neid ist.

\section{Ein Chef muss fähig sein, neben sich andere qualifizierte Fachleute wirken zu lassen, die in ihrem speziellen Bereich besser sind als er.}

\footnotetext{
* Zur besseren Lesbarkeit wird die männliche Form verwendet. Selbstverständlich bezieht sich das Gesagte auch auf die Chefärztin.
}

Korrespondenz: Prof. Dr. med. Marco Mumenthaler Neurologie FMH Witikonerstrasse 326 CH-8053 Zürich

mumenthaler33[at]bluewin.ch
Anforderungsprofil. Hier geht es im Besonderen um die Vorgesetztenfunktion im Bereich medizinischer Institutionen, also von Chefärzten. Sie müssen Garant dafür sein, dass an der von ihnen geleiteten Institution die Kranken fachlich und menschlich optimal betreut werden. In Universitätsspitälern sind die Chefärzte zugleich Direktoren von Kliniken, die unter anderem Verantwortung für die Forschung sowie auch für die Ausbildung von Medizinstudenten und - wie auch viele nicht-universitäre Chefärzte - von Assistenzärzten haben, also die künftige Ärztegeneration schulen müssen.

Aus diesen Funktionen ergeben sich bereits einige der Fähigkeiten, die der verantwortliche Chefarzt besitzen sollte:
Für die Jüngeren, die Assistenz- und Oberärzte, muss er nicht nur ein fachlich kompetenter Lehrer sein. Er muss für sie auch in anderer Hinsicht Vorbildfunktionen ausüben. Diese Jungen wollen nicht nur ihr Wissen und ihre Kenntnisse vermehren. Sie wollen auch erleben, wie man mit den Situationen im Rahmen des ärztlichen Alltags in der Patientenberatung und Patientenbetreuung umgeht: mit diagnostisch nicht gesicherten Krankheitsbildern, mit gemachten Fehlern, mit verzweifelten Angehörigen. Sie wollen lernen, wie man eine sorgfältige Anamnese aufnimmt, wie man Zusatzuntersuchungen gezielt einsetzt, wie man kritisch Einzelelemente ordnet und synthetisch zusammenfügt und deduktiv zu einer diagnostischen Beurteilung gelangt. Sie 
wollen demonstriert bekommen, wie man das therapeutische Vorgehen aus der Automatik einer starren Diagnose-Therapiesequenz in eine stets individuell angemessene Variante der Therapie überführt. Dies den Jungen mitzugeben, gehört zu den wichtigsten Aufgaben des Chefarztes. Er kann diese Aufgabe nur dann optimal erfüllen, wenn er neben der nötigen fachlichen und menschlichen Autorität für diese Funktionen genügend Zeit einsetzt. Dies geht logischerweise zulasten anderer Tätigkeiten, unter anderem von privatärztlichen Funktionen. Der Chefarzt ist auch der letztlich Verantwortliche für die optimale ärztliche Betreuung der Kranken. Er muss also für jeden Patienten bei Bedarf zur Verfügung stehen. Jeden hospitalisierten Patienten muss er mindestens einmal gesehen haben und muss für die Patienten in dem Masse verfügbar sein, wie es deren Problem erfordert. Daraus ergibt sich automatisch eine Schlussfolgerung in Bezug auf die Anzahl Patienten, für die ein einzelner Chefarzt verantwortlich sein kann. Die Grösse einer Klinik muss mit der Erfüllung der soeben umschriebenen Aufgaben vereinbar sein. Die Qualität des Wirkens muss immer stärker gewichtet werden als die Menge der zugeteilten Betten oder die Anzahl der Mitarbeiter. Diese letztliche ärztliche Verantwortung kann er nicht delegieren. Dennoch wird es so sein, dass ein qualifizierter verantwortlicher Oberarzt im Einzelfall entscheiden kann, wann er den Chefarzt zusätzlich zuziehen will.

Trotz der aus diesen Verpflichtungen sich ergebenden grossen zeitlichen Belastung des Chefarztes mit letztinstanzlichen ärztlichen Aufgaben ist es wünschenswert, dass er auch zeitlich beschränkt privatärztlich tätig ist. Nur in dieser Funktion übt er dauernd die eigentliche ärztliche Funktion vom primären Zugang zum Patienten, der Erarbeitung einer diagnostischen Beurteilung, der Anwendung einer Therapie und der Begleitung des Kranken aus. Diese Funktion soll er auch dauernd üben, denn er muss sie auch seinen Schülern vermitteln können. Der chen stellt und dessen Meinung und Massnahmen zu akzeptieren sind. Dies setzt im Rahmen des Teams, dem er vorsteht, Autorität voraus. Gewiss ist ihm bereits durch seine Position in der Spitalhierarchie formal Autorität zugesprochen worden. Diese stellungsbedingte Autorität ist jedoch auf die Dauer ungenügend. Er muss seine Autorität vielmehr durch fachliche Kompetenz, durch persönlichen Einsatz, durch Grosszügigkeit, durch Toleranz, durch Förderung seiner Mitarbeiter, durch vorbildlichen Umgang mit dem Patienten und durch menschliche Wärme festigen. Er muss überzeugen und nicht befehlen, muss jedoch in dem, was er letztlich beschliesst, klar, bestimmt und konsequent sich durchsetzen. Dies hat gelegentlich auch zur Folge, dass er zugunsten der Funktionstüchtigkeit des von ihm geleiteten Betriebes eigenen menschlichen Neigungen oder den Wünschen eines Mitarbeiters nicht entsprechen kann. So muss er z.B. unter Umständen eine Person in einer ihr nicht genehmen Funktion einsetzen oder auf die Weiterbeschäftigung eines Mitgliedes des Teams verzichten.

Im akademischen Krankenhaus hat der Chefarzt und Klinikdirektor noch zwei zusätzliche wichtige Verantwortungen. Er muss die Forschung in der eigenen Klinik fordern und fördern. Auch hier wie in der ärztlich-fachlichen Kompetenz wird er neben dem von ihm persönlich gepflegten Bereich andere Bereiche durch qualifizierte Mitarbeiter pflegen lassen müssen. Auch diesen gegenüber muss er sich in gleicher Weise, wie weiter oben für die klinisch-ärztlichen Mitarbeiter hervorgehoben wurde, verhalten. Da sehr oft Mitarbeiter sowohl klinische Verpflichtungen wie Forschungstätigkeiten ausüben, muss der Chefarzt dafür sorgen, dass durch entsprechende Entlastungen bzw. Freistellungen die Erfüllung beider Aufgaben zumutbar ist. Dass dies dennoch eine überdurchschnittliche Leistungsbereitschaft der Beteiligten voraussetzt, sollte trotzdem nicht zu unzumutbaren Belastungen oder zu einem Burn-out führen.

\section{Gelegentlich muss er zugunsten der Funktionstüchtigkeit des Betriebs eigene Neigungen oder Wünsche einzelner Mitarbeiter zurückstellen.}

Chefarzt wird dadurch immer in Kontakt mit der Realität der persönlichen Begegnung mit dem Patienten, für den er die volle persönliche Verantwortung übernimmt, sein. Die letztinstanzliche Tätigkeit als Chef ersetzt dies nicht, da hierbei viele wichtige Aspekte wegfallen.

Noch ein Wort zur Autorität. Autorität besteht nicht im Befehle erteilen. Der Chef ist in vielem der letztlich Verantwortliche, die letzte Instanz, derjenige, der verbindliche Entscheide fällt, die Wei-
An einer Universitätsklinik hat der Chefarzt und Direktor schliesslich auch Studenten zu unterrichten. Er darf nicht vergessen, dass er als Mitglied einer Medizinischen Fakultät Teil der Universität ist, deren Aufgabe unter anderem die Ausbildung der Studierenden ist. Je nach Unterrichtsmodus wird der Chefarzt somit entweder im Rahmen von Vorlesungen oder von Seminarien oder Gruppenunterricht seine Lehrtätigkeit ausüben. Und er hat wahrlich die Pflicht, sie gut auszuüben! Seine vornehmste Auf- 


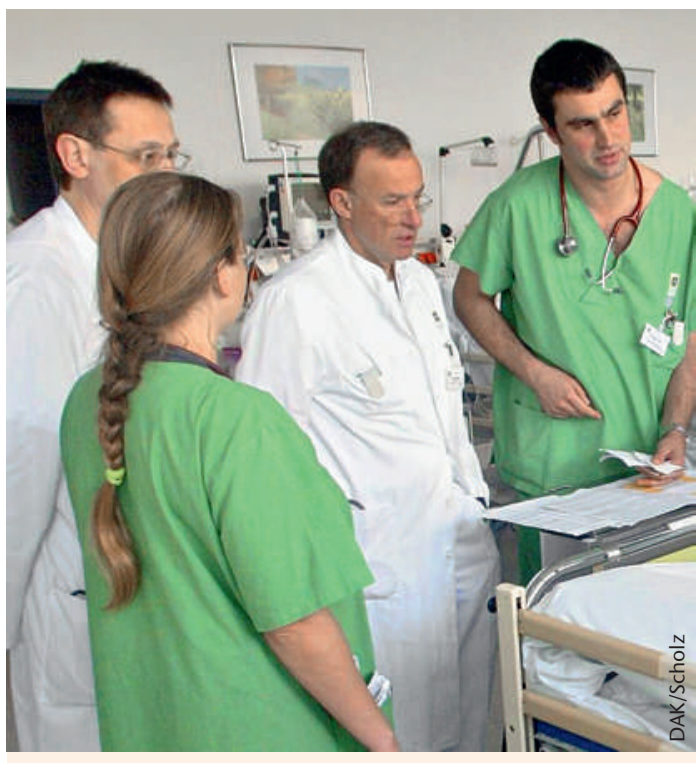

Fachliche Kompetenz, persönlicher Einsatz, Förderung der Mitarbeiter, Grosszügigkeit, Toleranz, vorbildlicher Umgang mit den Patienten, menschliche Wärme - all das macht die Autorität eines Chefarztes aus.

gabe ist nicht so sehr die, den Studierenden Fakten beizubringen. Diese können sich die Studenten auch aus Büchern, Videos oder durch E-Learning erwerben. Als Chefarzt hat er ihnen Vorbild zu sein. Er kann auch in einer Vorlesung beim Vorstellen eines Patienten den Studierenden zeigen, wie man menschlich auf die Kranken eingeht. Er kann ihnen sogar an Fällen zeigen, wie ethische Überlegungen unser Handeln mitbestimmen, er kann sie motivieren, unter Verzicht auf ein zusätzliches Einkommen auf unnötige Zusatzuntersuchungen zu verzichten.

Neben diesen fachbezogenen ärztlichen Aufgaben muss aber ein Chefarzt noch in einer ganzen Reihe anderer Bereiche wirken. Er muss jederzeit bereit sein, seinen Mitarbeitern und seinen Studenten persönlicher Berater auch in nicht berufsbezogenen Belangen zu sein. Er muss bereit sein, individuelle Probleme anderer mitzutragen. Er muss seine Mitarbeiter auf allen Stufen spüren lassen, dass er ihnen beizustehen bereit ist, wenn sie es wünschen. Dies wird allerdings nur dann gelingen, wenn der Chef- arzt frei von Hochmut ist, ein freundliches Naturell hat - was durchaus mit einer natürlichen Autorität verbunden sein kann - und auf die Mitarbeiter zugeht, ohne sich ihnen aufzudrängen. Dies alles setzt Einfühlungsvermögen, verfügbare Zeit und eine offene Türe voraus.

Das Erfüllen all dieser Aufgaben setzt die Fähigkeit voraus, die eigene Zeit gut einzuteilen, die Prioritäten richtig zu setzen, trotz konzentrierter Arbeitsweise und diszipliniertem Einsatz der Kräfte sich nicht zur unkontrollierten Hast verleiten zu lassen. Er muss auch delegieren können und den Mut haben, persönlich auf gewisse zeitlich belastende Aufgaben zu verzichten, selbst wenn diese verlockend sind.

Das bisher Gesagte ist schön und gut und mag auch wünschbar sein. Gibt es aber diese Übermenschen? Mir scheint aber nicht dies die wesentliche Frage zu sein. Die zwei wesentlichen Fragen sind vielmehr die folgenden:

- Kann man die oben aufgeführten Eigenschaften nicht durch entsprechendes Insichgehen fördern, anstreben oder durch entsprechende Veranstaltungen sogar schulen? Ich bin überzeugt, dass man dies kann.

- Kann man nicht bei der Wahl eines Chefarztes und insbesondere, wenn eine Fakultät einen Klinikdirektor wählt, auf die charakterlichen Besonderheiten und die menschlichen Qualitäten der Kandidaten achten? Gewiss ist dies schwieriger und aufwendiger als die quantitative und qualitative Auswertung durchgemachter Schulungen und veröffentlichter wissenschaftlicher Arbeiten. Eine Analyse des Verhaltens und der Wirkungsweise eines Kandidaten oder einer Kandidatin für Cheffunktionen an den bisherigen Wirkungsstätten gewissermassen vor Ort ist sehr wohl möglich, wenn auch zugegebenermassen aufwendig.

So, jetzt habe ich mir dieses Anliegen von der Seele geschrieben. Ich weiss, dass ich selber dem hier skizzierten Bild in vielem nicht entsprochen habe. Vielleicht wird aber der eine oder andere Chefarzt oder der eine oder andere für eine Chefarztwahl Verantwortliche diese Gedanken lesen und sich zu Herzen nehmen. 